University of Nebraska - Lincoln

DigitalCommons@University of Nebraska - Lincoln

Norman R. Simon Papers

Research Papers in Physics and Astronomy

$12-15-1973$

\title{
Multimodal Radial Pulsational Instability In A Prenova Model
}

V. K. Sastri

University of Nebraska-Lincoln

Norman R. Simon

University of Nebraska - Lincoln, nsimon@unl.edu

Follow this and additional works at: https://digitalcommons.unl.edu/physicssimon

Sastri, V. K. and Simon, Norman R., "Multimodal Radial Pulsational Instability In A Prenova Model" (1973). Norman R. Simon Papers. 33.

https://digitalcommons.unl.edu/physicssimon/33

This Article is brought to you for free and open access by the Research Papers in Physics and Astronomy at DigitalCommons@University of Nebraska - Lincoln. It has been accepted for inclusion in Norman R. Simon Papers by an authorized administrator of DigitalCommons@University of Nebraska - Lincoln. 
The Astrophysical Journal, 186:997-1005, 1973 December 15

(C) 1973. The American Astronomical Society. All rights reserved. Printed in U.S.A.

\title{
MULTIMODAL RADIAL PULSATIONAL INSTABILITY IN A PRENOVA MODEL
}

\author{
V. K. SASTRI AND Norman R. Simon \\ Behlen Laboratory of Physics, and Behlen Observatory, \\ University of Nebraska, Lincoln \\ Received 1973 June 7
}

\begin{abstract}
A prenova model, consisting of a white dwarf unstably burning a shell of accreted hydrogen, was tested for stability against radial pulsations. The pulsational analysis included terms due to thermal imbalance. The model was found to be pulsationally unstable in all of the modes examined, save one in which the thermal-imbalance integrals made the crucial damping contribution. An evaluation of some uncertainties in the present analysis is followed by a more general discussion in which it is concluded that multimodal radial pulsational instability can occur in a wide variety of thermally unstable, shell-burning stars. A brief discussion of some consequences of such instability, including its possible connection with the rapid blue variables, concludes the article.
\end{abstract}

Subject headings: instabilities - novae - pulsation - white dwarf stars

\section{INTRODUCTION}

It has been argued by Rose $(1967,1968)$ that stars experiencing thermal runaways in burning shells may, under certain circumstances, become unstable to nuclearenergized pulsations in the fundamental radial mode. The pulsational analysis employed by Rose was the standard quasi-adiabatic one (e.g., Schwarzschild and Härm 1959), and, as pointed out by Simon (1970), it neglected terms arising from the condition of thermal imbalance $\left(d S_{0} / d t \neq 0\right)$ in the models investigated. Subsequently, a procedure (also quasi-adiabatic) for taking account of such terms was suggested (Simon 1971), and was then applied to a number of polytropes (Simon 1971; Simon and Sastri 1972).

In the present work this same procedure is employed to treat radial pulsations in a prenova model of the sort described in Starrfield et al. (1972). This model (henceforth the S101 model) is found to be pulsationally unstable, not only in the fundamental but also in many higher modes, with the thermal-imbalance terms playing an important though (with one exception) not yet decisive role. Some of the implications of multimodal radial pulsational instability, especially as regards the rapid blue variables, are discussed in the final section.

\section{THE MODEL}

A model for novae presently favored by a number of investigators (e.g., Rose 1968; Starrfield $1971 a, b$ ) consists of a binary star system in which one member, a white dwarf, accretes hydrogen-rich matter from its companion. When enough matter has been accreted, the hydrogen begins to burn in a shell near the white dwarf's surface, a thermal runaway ensues, and eventually some or all of the accreted matter is ejected in an outburst whose observable effects constitute the nova phenomenon. Important details in this class of model differ with different investigators, but, as it is not our purpose in the present work to study the origins of novae, we shall make only such brief comments in this regard as seem appropriate. 
The model we treat here (S101) is a $1 M_{\odot}$ white dwarf including an outermost envelope consisting of $1.3 \times 10^{-3} M_{\odot}$ of accreted material. The envelope is burning hydrogen unstably via the CNO cycle, but the thermal runaway is in an early enough stage that hydrostatic equilibrium, as measured by the ratio $a / g$, is maintained to at : . worst a few parts in $10^{11}$. Virtually all of the nuclear energy production occurs in a relatively narrow convective shell situated at the base of the envelope. This shell is strongly in thermal imbalance.

The model had been evolved to its present state via a fully implicit, Lagrangian, hydrodynamic computer code (see Starrfield et al. 1972) whose requirements, as reflected in the model structure, posed some difficulties for the pulsational analysis. In the first place the white-dwarf core, which was uninteresting for the problem of Starrfield et al. (1972), but which nonetheless comprises nearly all the mass of the object, was not included. Although this core, as it turns out, also contributes virtually nothing to the pulsational stability integrals, integrations extending deeper than the shell were found to be necessary in order to determine the eigenvalues of the pulsation. Accordingly, a polytropic interior was fitted to the envelope model, thus furnishing the interior runs of $P, \rho$, and $r$ necessary for integrating the adiabatic pulsation equations. The index of the appropriate polytrope turned out to be $n=1.66$, not too different from the value $n=1.5$ corresponding to a fully degenerate, nonrelativistic gas. Some model characteristics are given in table 1.

A second problem with the pulsational analysis had to do with the relatively crude zoning in the outer layers of the S101 envelope. This sort of zoning, while appropriate for the demands of a large hydrodynamic code, can cause some difficulties in integrating the pulsation equations, particularly when the higher modes are involved. To remedy these difficulties, quadratic interpolation was performed among the given points in the envelope model, thus providing a denser grid of envelope physical variables (i.e., a smaller step size) against which the pulsation equations could be integrated.

Nuclear burning in the S101 model is quite unusual in that the CNO cycle is far out of equilibrium and $\beta^{+}$-unstable nuclei begin to accumulate in the peak burning region and are subsequently mixed throughout the convective shell. The effect of this mixing is to extend the nuclear energy generation outward into regions that would normally be too cool for the equilibrium CNO cycle to operate effectively. Furthermore, the decay of these nuclei provides an energy source independent of temperature and density and thus not susceptible to influence by the pulsation nor available as a source or sink of pulsational energy. For these reasons the following procedure was adopted. The part of the nuclear energy emitted via $\beta$-decays, $\epsilon_{\beta}$, was subtracted at each point in the shell from the total nuclear energy generation rate $\epsilon$ given in the model, the difference $\epsilon_{e f f}=\epsilon-\epsilon_{\beta}$ being taken as the appropriate quantity to enter calculation of the energizing integral in the pulsation analysis. Since convection mixes the $\beta^{+}$-unstable nuclei uniformly through the shell, $\epsilon_{\beta}$ is constant and was

TABLE 1

Model Characteristics fOR S101

\begin{tabular}{cccc}
\hline \hline Variable & Surface & Shell & $\begin{array}{c}\text { Outer Edge of } \\
\text { Convective Region }\end{array}$ \\
\hline $\mathrm{M}_{r} / M_{\odot} \ldots \ldots$ & 1.000 & 0.9987 & 0.9999 \\
$\log r(\mathrm{~cm}) \ldots \ldots$ & 8.905 & 8.853 & 8.886 \\
$\log \left(\mathrm{L}_{r} / L_{\odot}\right) \ldots$ & -1.776 & 6.945 & 4.903 \\
$\log T \ldots \ldots$ & $\ldots$ & 7.569 & 7.072 \\
$\log \rho \ldots \ldots$ & $\ldots$ & 4.042 & 3.277 \\
\hline
\end{tabular}


evaluated at the cool edge of the shell where proton- and alpha-capture reactions: have already become negligible as an energy source (i.e., at a point where $\epsilon=\epsilon_{\beta}$ ).' ' ' The quantity $\epsilon_{\beta}$ amounts to less than 2 percent of the peak nuclear energy generation, rate deep in the shell. Finally, for the purposes of the pulsational analysis, the effective : . energy generation rate was fitted to the formula.

$$
\epsilon_{\text {eff }}=K \rho T^{v_{\text {eff }}}
$$

with $K$ and $\nu_{\text {eff }}$ constant, the latter having the value 13.1. The error in this fit was totally negligible throughout the region where nuclear reactions make any appreciable contribution to pulsational energizing.

\section{THE PULSATIONAL ANALYSIS}

The pulsational analysis employed in this work is based on the so-called "energy method" (e.g., Ledoux 1958), and has been discussed in detail by Simon (1970, 1971) and Simon and Sastri (1972). In this approach the pulsational stability of a given mode is determined by evaluation of the sum of a number of integrals:

$$
\begin{aligned}
\frac{L p}{L}=\int_{0}^{M} \frac{\left(\Gamma_{3}-1\right)}{L} \rho_{* 1} \delta \epsilon d m-\int_{0}^{M} \frac{\left(\Gamma_{3}-1\right)}{L} \rho_{* 1} \frac{\partial}{\partial m}\left(\delta L_{r}\right) d m \\
\quad-\int_{0}^{M} \frac{\left(\Gamma_{3}-1\right)^{2}}{L}\left(\epsilon-\frac{\partial L_{r}}{\partial m}\right)\left(\rho_{* 1}\right)^{2} d m \\
\quad+\int_{0}^{M} \frac{\rho^{2}}{2 T} \frac{\partial^{3} U}{\partial S \partial \rho^{2}} \frac{1}{L}\left(\epsilon-\frac{\partial L_{r}}{\partial m}\right)\left(\rho_{* 1}\right)^{2} d m+2 \int_{0}^{M} \frac{\left(\Gamma_{3}-1\right)}{L}\left(\epsilon-\frac{\partial L_{l}}{\partial m}\right) \rho_{* 0} d m,
\end{aligned}
$$

where $\delta \epsilon$ and $\delta L_{r}$ are the linear variations in the nuclear energy generation rate $\epsilon_{\text {eff }}$ and in the luminosity, respectively; and, in accordance with the notation introduced in Simon $(1972 b), \rho_{* 1}$ is the linear relative density variation and $\rho_{* 0}$ the surviving time-averaged (over the pulsation period) relative density variation. (Note that these were called $\rho_{*}$ and $\langle\delta \rho / \rho\rangle$, respectively, in Simon 1971.) Labeling these integrals in order, we have

$$
L p / L=N_{1}+I_{1}+I_{2}+J_{2}+K_{2} \text {. }
$$

The first two terms on the right-hand side constitute the usual quasi-adiabatic evaluation; the last three terms are due to thermal imbalance. A positive value of $L p / L$ indicates pulsational instability; a negative value, stability.

Expressions for $\rho_{* 1}$ and $\rho_{* 0}$ are given explicitly in Simon (1971). For the present model we have taken $\Gamma_{3}-1=0.667$, and $\left(\rho^{2} / 2 T\right)\left(\partial^{3} U / \partial S \partial \rho^{2}\right)=-0.111$. These values are appropriate (Simon 1970) both for an ideal gas with negligible radiation pressure and for a fully degenerate nonrelativistic electron gas. All relevant regions of the model we are treating fall into these thermodynamic classifications.

Table 2 gives the square of the dimensionless angular frequency, $\omega^{2}$, for the fundamental and the next seven modes, as well as the values of various pulsational quantities at the surface of the model and in the shell region of peak nuclear burning. The quantity $l_{* 1}(\mathrm{rad})$ is just the relative first-order variation of the radiative luminosity, calculated from linearization of the equation of radiative transport and assuming a constant opacity; $l_{* 1}$ (turb) is the relative variation of the convective luminosity and is given by

$$
l_{* 1}(\text { turb })=2 x_{*}+\Gamma_{3} \rho_{* 1}
$$


TABLE 2

Pulsational Characteristics for S101*

\begin{tabular}{|c|c|c|c|c|c|c|c|c|}
\hline $\begin{array}{l}\text { Mode } \ldots \ldots \ldots \\
\omega^{2} \ldots \ldots \ldots\end{array}$ & $\begin{array}{c}0 \\
3.86\end{array}$ & $\begin{array}{r}1 \\
15.6\end{array}$ & $\begin{array}{r}2 \\
27.2\end{array}$ & $\begin{array}{r}3 \\
41.1\end{array}$ & $\begin{array}{r}4 \\
64.0\end{array}$ & $\begin{array}{r}5 \\
85.3\end{array}$ & $\begin{array}{r}6 \\
109\end{array}$ & $\begin{array}{r}7 \\
141\end{array}$ \\
\hline $\begin{array}{l}\text { Surface: } \\
\quad \rho_{* 1} \ldots \ldots \\
\quad l_{* 1}(\mathrm{rad}) \\
u_{*} \ldots \ldots \\
\rho_{* 0} \ldots \ldots\end{array}$ & $\begin{array}{r}-4.72 \\
-8.57 \\
1.53 \\
-2.07\end{array}$ & $\begin{array}{l}-11.8 \\
-27.4 \\
4.01 \\
-20.4\end{array}$ & $\begin{array}{c}-18.7 \\
-45.9 \\
6.78 \\
-55.2\end{array}$ & $\begin{array}{r}-27.0 \\
-68.1 \\
10.1 \\
-119\end{array}$ & $\begin{array}{c}-40.8 \\
-105 \\
15.6 \\
-274\end{array}$ & $\begin{array}{c}-53.6 \\
-139 \\
20.8 \\
-474\end{array}$ & $\begin{array}{c}-68.1 \\
-177 \\
26.9 \\
-768\end{array}$ & $\begin{array}{c}-87.2 \\
-228 \\
35.2 \\
-1260\end{array}$ \\
\hline 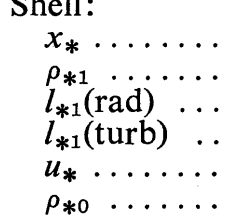 & $\begin{array}{l}+0.832 \\
-3.62 \\
-6.12 \\
-4.37 \\
1.06 \\
-1.36\end{array}$ & $\begin{array}{c}+0.330 \\
-4.34 \\
-9.19 \\
-6.57 \\
0.487 \\
-3.14\end{array}$ & $\begin{array}{l}+0.082 \\
-3.16 \\
-6.54 \\
-5.11 \\
0.121 \\
-2.08\end{array}$ & $\begin{array}{l}-0.036 \\
-1.27 \\
-2.00 \\
-2.19 \\
0.034 \\
-0.651\end{array}$ & $\begin{array}{c}-0.047 \\
+0.693 \\
+2.22 \\
+1.06 \\
0.031 \\
-0.216\end{array}$ & $\begin{array}{l}-0.011 \\
+0.920 \\
+2.04 \\
+1.51 \\
0.010 \\
-0.214\end{array}$ & $\begin{array}{r}+0.014 \\
+0.291 \\
+0.093 \\
+0.512 \\
0.006 \\
-0.072\end{array}$ & $\begin{array}{r}+0.011 \\
-0.440 \\
-1.36 \\
-0.711 \\
0.005 \\
-0.067\end{array}$ \\
\hline
\end{tabular}

* Pulsational quantities normalized to $x_{*}($ sur $)=1.0$.

an expression taken from Boury, Gabriel, and Leboux (1964), according to a scheme in which convection does not adapt to the pulsation. For the convective region in consideration here, the ratio of the convective to the sound velocity nowhere exceeds about 0.005 . Finally, $x_{*}$ is just the relative linear radius variation and $u_{*}$ the surviving time-averaged component (Simon 1971; Simon and Sastri 1972). All pulsational amplitudes are normalized to a value $x_{*}($ sur $)=1.0$.

The pulsational stability of the various modes is indicated in detail in table 3 . The periods are what one expects for a white-dwarf configuration, and, as can be seen, all of the modes tested, save one (the fourth overtone), turn out to be unstable.

The behavior of the integral $I_{1}$ is interesting here since for the first four modes it does not fulfill its traditional role as a damping term but instead contributes to the pulsational energizing. We may understand this behavior with the aid of an integration by parts, putting the integral into the form

$$
I_{1}=-\left.\left(\Gamma_{3}-1\right) f \rho_{* 1} l_{* 1}\right|_{\text {sur }}+\int_{0}^{M}\left(\Gamma_{3}-1\right) f l_{* 1} \frac{\partial}{\partial m}\left(\rho_{* 1}\right) d m,
$$

where $f=L_{r} / L$.

Since $f$ (shell) $/ f$ (sur) $=5.26 \times 10^{8}$, while $l_{* 1}$ and $\rho_{* 1}$ fall off (even for the highest modes calculated) by at most a factor $\sim 10^{2}$ from surface to shell, one sees that,

TABLE 3

Results of Pulsational Stability Analysis

\begin{tabular}{|c|c|c|c|c|c|c|c|c|}
\hline \multirow[b]{2}{*}{ PARAMETER } & \multirow[b]{2}{*}{0} & \multicolumn{6}{|c|}{ MODE } & \multirow[b]{2}{*}{7} \\
\hline & & 1 & 2 & 3 & 4 & 5 & 6 & \\
\hline $\begin{array}{l}\begin{array}{l}\text { Period } \\
\text { (seconds) }\end{array} \\
N_{1}\left(10^{9}\right) \ldots \ldots \\
I_{1}\left(10^{9}\right) \ldots \ldots \\
I_{2}\left(10^{9}\right) \ldots \ldots \\
J_{2}\left(10^{9}\right) \ldots \ldots \\
K_{2}\left(10^{9}\right) \ldots \ldots \\
\mathbf{L}_{p} / L\left(10^{9}\right) \ldots \ldots \\
\text { e-folding time }\end{array}$ & $\begin{array}{c}\quad 6.34 \\
90.2 \\
+0.538 \\
-7.81 \\
-1.95 \\
-2.37 \\
+78.6\end{array}$ & $\begin{array}{c}3.15 \\
135 \\
+4.88 \\
-15.8 \\
-3.95 \\
-7.62 \\
+112\end{array}$ & $\begin{array}{c}2.39 \\
76.1 \\
+6.69 \\
-13.8 \\
-3.45 \\
-7.66 \\
+57.9\end{array}$ & $\begin{array}{c}1.94 \\
15.0 \\
+4.01 \\
-7.66 \\
-1.91 \\
-4.84 \\
+4.55\end{array}$ & $\begin{array}{l}1.56 \\
2.74 \\
-0.576 \\
-1.88 \\
-0.470 \\
-1.49 \\
-1.68\end{array}$ & $\begin{array}{l}1.35 \\
6.09 \\
-0.609 \\
-0.703 \\
-0.176 \\
-0.639 \\
+3.96\end{array}$ & $\begin{array}{l}1.19 \\
1.09 \\
-0.103 \\
-0.437 \\
-0.109 \\
-0.414 \\
+0.027\end{array}$ & $\begin{aligned} & 1.05 \\
& 1.13 \\
&-0.421 \\
&-0.310 \\
&-0.077 \\
&-0.297 \\
&+0.025\end{aligned}$ \\
\hline$\left(10^{7} \mathrm{~s}\right) \ldots \ldots$ & +150 & +4.6 & +0.71 & +14.0 & -19 & +1.7 & +350 & +140 \\
\hline
\end{tabular}


contrary to the familiar case, the integrated term in the above expression is negligible, and the sign of $I_{1}$ will thus be determined by the sign of the product $l_{*_{1}} \partial\left(\rho_{* 1}\right) / \partial m$ in the narrow shell region of peak luminosity. Because the sign of this product is itself determined by the specific form taken by the individual eigenfunctions $l_{* 1}$ and $\rho_{* 1}$.! for each mode, it seems only possible to say that in a model such as this one, $I_{1}$ may play either an energizing or a damping role for a given mode, an actual calculation of the eigenfunctions involved being necessary to determine its sign. The values of $I_{1}$ given in table 3 are all calculated by taking $l_{* 1}=l_{* 1}(\mathrm{rad})$. We shall say more about this later.

One may compare the results of the present work with those of Rose $(1967,1968)$ in which $I_{1}$ is a damping term for all models evaluated. The reason for this discrepancy lies in the comparative mildness of the thermal instability in Rose's models, the ratio $f$ (shell) $/ f$ (sur) never exceeding $\sim 10^{4}$.

Returning to table 3, one observes that for the first few modes the nuclear energizing $N_{1}$ far outweighs all other terms, while for the higher modes the balance is closer. In all cases the terms arising from thermal imbalance $\left(I_{2}, J_{2}\right.$, and $\left.K_{2}\right)$ are negative, in agreement with Simon and Sastri (1972), and their combined effect always provides the major portion of the damping. For the one stable mode the thermal-imbalance terms are themselves large enough to provide the necessary damping even though $I_{1}$ is also a negative term. The fact that this mode is stable is due to the comparative smallness of $N_{1}$ occasioned by the fact that a node in $\rho_{* 1}$ lies relatively close to the peak region of nuclear burning.

Finally, one may note, again from table 3, an apparent trend toward stability appearing in the two highest modes. Because of this we have calculated one more mode, the eighth overtone, which turns out to be more unstable than the previous two, exhibiting no tendency that we can notice toward stabilization.

\section{DISCUSSION}

We wish to begin this section by pointing out that the conclusions we shall eventually draw from this work will be cautious and tentative ones; it is not our purpose to offer the pulsational properties of this particular model as specific explanations for various observed phenomena whose relationship to radial pulsations may or may not eventually be demonstrated.

With the above in mind we shall proceed with an attempt to evaluate some of the inadequacies in the calculations described in $\S$ II and III. First with regard to the fitting of a polytropic core to the envelope model S101, we consider that the difference between our results and those which could be obtained with a true evolutionary white-dwarf interior would consist merely in small changes in the eigenvalues of the pulsation - the eigenfunctions and thus the stability integrals would remain essentially unchanged in the qualitative and semiquantitative senses of whether one has stability or instability and in the rough pulsational rise times of the various modes.

The above evaluation also applies, we believe, to the effects of interpolation in the envelope model. No doubt the lack of a careful treatment of the surface layers of the model renders the pulsational analysis somewhat crude, particularly with regard to the higher modes; however, we can detect no systematic error in this, and no reason to call into question the conclusion that many higher modes appear to be pulsationally unstable.

Similarly our treatment of the pulsational effects of nuclear burning does not seem to contain inherent problems. The use of the quantity $\epsilon_{\mathrm{eff}}$ to eliminate the $\beta$-decays in evaluating $N_{1}$ is quite consistent and logical. The thermal-imbalance integrals, on the other hand, were calculated employing the true nuclear energy-generation rate $\epsilon$. Since it is the peculiarity of this model that the energy generation extends somewhat 
farther out toward the surface than would be the case if the CNO cycle were in equilibrium, one might think that the thermal-imbalance damping would be somewhat ' ' enhanced compared with a more ordinary case. However, as one begins dealing with the higher modes, location of a source or sink of pulsational energy somewhat closer : . to the surface loses to a great extent its usual advantage, since the shell is already in a region interior to the precipitous initial fall-off of the eigenfunctions, and the amplitude of a given mode in the shell region depends mainly on where the nearest node happens to appear.

A more serious problem involves our evaluation of the integral $I_{1}$ using the radiative amplitude $l_{* 1}(\mathrm{rad})$, since we have already noted that virtually the entire contribution to $I_{1}$ comes from the shell region which is convective. In the S101 model the approximation has been made that within the convective region all of the flux is carried by convection, while outside of it none is. The result is, of course, a discontinuity which does not allow a proper treatment of $I_{1}$ in this region. For purposes of comparison we have calculated $l_{* 1}($ turb) at the shell, according to equation (1). As one can see from table 2 , in most of the cases $l_{* 1}(\mathrm{rad})$ and $l_{* 1}($ turb) have comparable values, although in one case in particular (overtone 6) the difference is considerable. Of course, neither of these quantities is itself the proper one to insert into $I_{1}$ and it is possible that our use of $l_{* 1}(\mathrm{rad})$ underestimates the value of $I_{1}$, a quantity which begins to become important as we go to higher modes. Thus, for example, a marginally unstable mode like the sixth overtone could possibly be stabilized by a stronger $I_{1}$. However, in view of the above noted facts that the sign itself of $I_{1}$ shows no definite observable trend and that this integral does not seem to make a dominant contribution, at least for most of the modes calculated, once more we believe that there is no systematic error here that would negate our finding of many unstable modes.

By far the most serious uncertainty in the present work lies in our evaluation of the pulsational effects of thermal imbalance. No investigator has yet demonstrated conclusively that the energy approach initiated by Thomas (1931), elaborated in Ledoux (1958), and further developed by Simon $(1970 ; 1971)$ and Simon and Sastri (1972) is the correct one. A somewhat different approach (Kato and Unno 1967), but still based on an energy method, was applied to a polytrope of index $n=1.5$ by Okamoto (1967). His results for the thermal-imbalance contribution agreed to within about 4 percent with those reported in Simon and Sastri (1972) for the same polytrope. However, in a very recent work (Cox, Hansen, and Davey 1973) in which a method involving direct linearization of the pulsation equations (e.g., Ledoux 1963) was employed, a far different result was obtained for the $n=1.5$ model, the discrepancy with the earlier results amounting to 30 or 40 percent, and the only agreement being in sign. A discussion of these differences is given in Cox et al. (1973).

Furthermore, even if one takes the energy approach (Simon 1971) to be correct, a problem still exists regarding the proper manner in which to evaluate the integral $K_{2}$, a quantity which depends upon pulsational terms which are intrinsically of second order. The iterative technique for the calculation of such terms (Simon 1972a) depends upon the assumption that linear analysis of a given model has revealed one and only one unstable mode. In the case of thermal imbalance, however, the "linear analysis" itself involves (via $K_{2}$ ) terms intrinsically of second order. Were we to perform this analysis disregarding $K_{2}$, we would still find many unstable modes, calling into question our subsequent method of evaluating $K_{2}$. Given these circumstances, we have proceeded in the only manner presently possible-namely, calculating the stability of each mode independently of the others, including evaluation of the integral $K_{2}$. Clearly, if we have made a substantial error in calculation of the pulsational effects of thermal imbalance, either because of deficiencies in the energy method or because of improper evaluation of $K_{2}$, then systematic changes in our 
results are possible, particularly and most importantly as regards the higher modes.

The development of a proper, proven, and generally agreed upon solution to the thermal-imbalance problem represents, in our opinion, an important frontier in the theory of stellar pulsations.

\section{CONCLUSION AND POSSIBLE OBSERVATIONAL CONSEQUENCES}

In this section it will be our purpose not so much to discuss the results of our calculations on the specific model S101, but rather to speak in a more general way concerning radial pulsations in stars burning nuclear fuel unstably in a thin shell near the stellar surface.

The model we have evaluated has, in addition to some of the properties discussed in an earlier section, yet another quite important characteristic-namely, its extremely rapid evolution time. In less than 7 hours, the peak rate of nuclear energy generation will have risen to a value of the order of $10^{13} \mathrm{ergs}^{-1} \mathrm{~s}^{-1}$, an increase by a factor greater than 100 . Shortly thereafter the nova outburst will begin. Since a glance at table 3 shows that the shortest pulsational $e$-folding time calculated for any of our modes is a few months, it becomes clear that the rise of "soft" pulsations here is impossible. Thus for a model such as this one, soft pulsations cannot be evoked as a medium for storing nova outburst energy (e.g., Rose 1968), nor can the rise of these pulsations affect in any way the evolution of the model toward outburst.

Considerably more interesting from our point of view are thermally unstable shellburning models, in which the instability is less violent and the evolution times longer, and which perhaps never reach the ferocity necessary to trigger the nova phenomenon. On the basis of the present work, of an investigation reported by us earlier (Simon and Sastri 1973), and of previous papers by Rose (1967; 1968) and Vauclair (1972), and taking into account the uncertainties raised in the previous section, it is our considered judgment that multimodal radial pulsational instability can occur in a wide variety of thermally unstable shell-burning stars.

In this connection, we believe it probable that Rose $(1967,1968)$ would have found pulsational instability in higher modes than the fundamental in a number of his models, had he looked for it; and that Vauclair (1972), who does not make it clear whether a thermal runaway is present or not in his hydrogen-accreting white dwarfs, may also have found pulsational instability beyond the first overtone.

Let us briefly consider, then, a white dwarf which has accreted a hydrogen shell (or perhaps a single star which has a helium shell) and burns it unstably in such a way that a number of radial pulsational modes become excited. We shall here assume that $e$-folding times for the pulsations are short enough compared to evolution times (as is marginally achieved for the fundamental mode in models of Rose 1967, 1968, and should be more easily achieved for higher modes) such that the amplitudes may grow to finite size. The unstable modes will now presumably interact, coupling in some time-dependent manner, such that energy is constantly flowing out of some modes and into others. The time scale for such coupling is not known, the only reasonable estimates presently available being the linear rise times of the various modes. It is possible that such a system eventually relaxes into a steady-state configuration (as seems to be the case with certain Cepheid-type pulsators which are linearly unstable in more than one mode [Christy 1966]) in which each mode on the average neither gains nor loses energy; on the other hand, this sort of relaxation may never be achieved within evolutionary time scales (the possibility of such a condition existing is made plausible by the behavior of the "mixed mode" model in Keeley [1970]). Existing literature on coupled nonlinear oscillations (e.g., Jackson 1963) does not seem adequate to provide insight into the stellar problem.

Furthermore, for those cases in which amplitudes are large and $e$-folding times reasonably rapid, one may also be confronted with a sort of pulsational backlash in 
which the secular evolution of the star is itself influenced by the pulsations, thus rendering inaccurate evolutionary calculations which ignore the pulsational instability. This is another subject which has received little attention in the literature.

We shall close this article with a few remarks regarding the so-called rapid or :.! ultrashort-period blue variables. A brief summary of some of the literature concerning these objects appears in Osaki and Hansen (1973). The stars lumped together under this classification actually constitute an extremely diverse menagerie ranging from old novae like DQ Her (Warner et al. 1972), to dwarf novae like Z Cam (Robinson 1973), to X-ray sources such as Sco X-1 (Robinson and Warner 1972), to white-dwarf-type objects like R548 (Lasker and Hesser 1971), etc. If they all have anything in common, it seems to consist of a rapid flickering on time scales ranging from a few tens of seconds (e.g., Z Cam) up to perhaps 1000 seconds (e.g., HL Tau-76; Warner and Robinson 1972). Furthermore, the kinds of variability run from the quite regular 71-s period of DQ Her, to the multiperiodic behavior of Z Cam and HL Tau-76, to what appears to be an aperiodic jittering in the light of Sco X-1. In addition the amplitudes of variation in these objects show a wide diversity, with the variation of $\mathrm{Z}$ Cam being only $\sim 0.001 \mathrm{mag}$ whereas HL Tau-76 attains an amplitude as great as 0.4 mag (Warner and Robinson 1972).

A number of models for the rapid blue variables have appeared in the literature, with recent emphasis on nonradial oscillations as the seat of the variability (e.g., Harper and Rose 1970; Warner and Robinson 1972; Osaki and Hansen 1973). It is not our purpose here to analyze or discuss any of these models. However, on the basis of the present work, we would like to emphasize that radial pulsations may well have a role to play in some of the ultrashort-period variables, either as a direct source of the variability or as a contributing mechanism.

Although the radial pulsation periods found for S101 are too short to match even the quickest of the observed periodicities (e.g., 16-19 s for Z Cam [Robinson 1973]; 24-25 s for CN Ori [Warner and Robinson 1972]), a number of the models of Rose $(1967 ; 1968)$ show periods of just this order. Furthermore, in the case where two or more higher modes with extremely short $(\lesssim 1 \mathrm{~s})$ and close periods become unstable, detection of the underlying periodicities becomes more and more difficult while the possibility emerges that much longer modulation time scales may arise.

A still more speculative proposition is that where the instability is multimodal and unrelaxed (in the sense of a constant energy transfer among modes), the time scale one observes might, again, not be the underlying periodicity but rather that involved in energy flow from one mode to another. Since any in real physical (i.e., nonlinear) pulsation, the mean stellar parameters (the average being taken over a pulsation period) are amplitude and shape dependent (e.g., Simon 1972b), a transfer of pulsational energy from one mode to another will in general include a slow shift in mean properties of the star-for example, in the light. Whether or not such shifts could take place in times as short as, say, minutes, corresponding to the variability of an object like HL Tau-76, is a question we are unable at present to attack. Pulsational analysis, both linear and nonlinear, of a diverse collection of shell-burning models seems advisable at the present time if we are to get a better idea of the possible role played by radial pulsations in the ultrashort-period stars.

We are grateful to Dr. S. Starrfield both for kindly providing us with the model S101 and for useful discussions.

\section{REFERENCES}

Boury, A., Gabriel, M., and Ledoux, P. 1964, Ann. d'Ap., 27, 92.

Christy, R. F. 1966, Ann. Rev. Astr. and Ap., 4, 353.

Cox, J. P., Hansen, C. J., and Davey, W. R. 1973, Ap. J., 182, 885.

Harper, R. V. R., and Rose, W. K. 1970, Ap. J., 162, 963. 
Jackson, E. A. 1963, J. Math. Phys., 4, 686.

Kato, S., and Unno, W. 1967, Pub. Astr. Soc. Japan, 19, 1.

Keeley, D. A. 1970, Ap. J., 161, 657.

Lasker, B. M., and Hesser, J. E. 1971, Ap. J. (Letters), 153, L151.

Ledoux, P. 1958, in Handbuch der Physik, 51, 605. 1963, in Star Evolution, ed. L. Gratton (New York: Academic Press), pp. $394 \mathrm{ff}$.

Okamoto, I. 1967, Pub. Astr. Soc. Japan, 19, 384.

Osaki, Y., and Hansen, C. J. 1973 (preprint).

Robinson, E. L. 1973, Ap. J., 180, 121.

Robinson, E. L., and Warner, B. 1972, M.N.R.A.S., 157, 85.

Rose, W. 1967, Ap. J., 150, 193.

. 1968, ibid., 152, 245.

Schwarzschild, M., and Härm, R. 1959, Ap. J., 129, 637.

Simon, N. R. 1970, Ap. J., 159, 859.

1971, ibid., 164, 331.

1972a, Astr. and Ap., 21, 45 1972b, ibid., p. 51.

Simon, N. R., and Sastri, V. K. 1972, Astr. and Ap., 21, 39. 1973, Bull: $A A S, 5,15$.

Starrfield, S. 1971 a, M.N.R.A.S., 152, 307. $1971 b$, ibid., 155, 129.

Starrfield, S., Truran, J. W., Sparks, W. M., and Kutter, G. S. 1972, Ap. J., 176, 169.

Thomas, L. H. 1931, M.N.R.A.S., 91, 122, 619.

Vauclair, G. 1972, Ap. Letters, 12, 17.

Warner, B., Peters, W. L., Hubbard, W. B., and Nather, R. E. 1972, M.N.R.A.S., 159, 321.

Warner, B., and Robinson, E. L. 1972, Nature Phys. Sci., 239, 2. 